\title{
The equivalence of SpaceTime curvature and Information wave function Entropy: a link between quantum mechanics and general relativity in the Universe and in the Brain
}

\author{
Hind Zaaraoui
}

\begin{abstract}
This work proposes a theory about how reaction responses to an incoming Information are created in Brain and similarly how the gravity or spacetime curvature of Universe are created with a same mathematical model that may conciliate quantum mechanics and general relativity in these two spaces.

In the brain: an external incoming signal information being initially modulated by recepetor neurons (in eyes, ears...) travels through the neighboring neurons that are linked to the excited receptor neurons. These neuronal paths have been created thanks to the past personal experiences. Potential reaction responses are subsequently created thanks to a final deformed signal in the motor neurons by all the correlated neural paths.

The total deformation at each neuron is created by different incoming paths and their structures: geometry of paths, respective neurotransmitters (inhibitors, exciters...), and of course the existence or not of the signal and its frequency coming from each path. Using path Integrals theory, we compute the probability of existence of the signal-Information or the potential reaction to the incoming information at each neuron. But we would like to know how much the signal-Information has been distorted between two neighboring linked neural points including if it arrives or not to the neigboring neurons. We thus propose an entropy of Information coming from each path (called Time path) to a particular neuron and we demonstrate that this entropy is equivalent to TimeSpace curvature in the Brain. Therefore the created reaction to the initial Incoming information is the heaviest and the nearest or in other words the most correlated reaction to the initial incoming Information.

Similarly in the Universe, the evolution of elementary particles in quantum mechanics can be described by path Integrals formulation using the action $S$ of each path. Thanks to high density of paths next to highly massive objects, the probability of existence of another less massive object would be higher next to the first object. Finally the SpaceTime curvature is demonstrated to be equivalent to the entropy of these paths.
\end{abstract}

\section{INTRODUCTION}

Our brain integrates all external and internal input signal information to give an output which is an internal or/and external signal reaction (reminding, thinking, speaking, laughing etc.) thanks to the complex links between neurons.

In the Brain, the information disseminators are neurons and all their structures including their axons, dendrites, synapses with neurotransmitters (inhibitors and excitors). The signal unit, which is called action potential in the brain, coming from a particular neuron with its particular synapses and neurotransmitters, propagates to the linked neighboring neuron thanks to the neurotransmitters which will ensure the circulation of the deformed (or not) signal information until the neighboring neuron, see for example Fig.1 and [1], [2], [3].

If the neurotransmitters are inhibitors then the created signal in the dentrites are reducing the final computed signal amplitude which may eliminate totally the signal propagation. The signal does no more exist in space nor in time. On the contrary if the neurotransmitters are excitors, the created signals in the dendrites enhances the computed signal which may ensure the continuation of the signal propagation distorted or not. From the dendrites to the neighboring neuron soma, all the incoming signals (inhibitors or excitors) ar summed in time and space [4], [5]. If the resulted amplitude exceeds a certain threshold then a new deformed signal is recreated in the studied neuron and retransmistted to the nearest linked neurons ([6] Fig. 1.

In this article we show that this signal/information deformation induced by all the neuron system axon, synpases, dendrites, soma ... is what we call the spacetime curvature at the neuron point and the resulted signal is the new distorted resulted information signal.

In the Universe, an Information, or what we call the signal-Information (set of action potentials) in the brain, can be an object, a particule, an energy ... If the information still exist, i.e it can be detected, travels inevitably in time form $t$ to $t+1$ and may spread also in space if a correlated external additional information exists: interactions, forces...od correlation.

To see the similarity with the brain function where signal-Information propagate, the inevitable time travel between times $t$ and $t+1$ of any created signal Information from a neuron soma is its travel in the following path in synpases (if there are still neurotransmitters left), dendrites and neuron soma. All this part synapses, neurotransmitters, dendrites and neuron 
soma is what we will call "Time" in the brain. The signal deformed by the Time arrives to the neighboring neuron soma which may be retransmitted or not, depending on the amplitude of the additionned arrived excitatory and inhibitory signals. Space is hence the axonic cone and axon where a potential signal may be created, or not, and retransmitted automatically in Time. See for example figure Fig. 1 for a better understanding of the structure of a neuron. One can see that Space and Time cannot be disconnected: a neuron axonal cone and axon exists if its dendrites, synapses, neurotransmitters and neuron soma exist and reciprocally.

Thanks to the existence of many neighboring neurons and to their following Time paths, an Information, more or less deformed, created in a given neuron at the instant $t$ may have the possibility of being present at the instant $t+1$ in all these neighboring neurons at the same time with different probability. Each of these neighboring neurons may create in the future a possible reaction: this what we can call Reaction state superposition.

The way the brain works is similar on how our universe work. There is the Space with its three dimensions and Time with unknown number of dimensions that one may not easily break their link... Space is what we can see in the known three dimensions. It is all the locations where any quantum particles could be created or recreated.In the humain behaviors these fields are all the type of reactions that a human can have: mouth field from laughing to having a political discussion, hands field from writting to scratching etc.

Time is maybe these undectable dimension $(s)$ that exist and can be seen in any kind of evolution and change of anything in universe (motions, interactions, disintegration, ...). What happens in this dimension is comparable to what partially happens in quantum "Vacuum" defined in Quantum Field Theory (QFT) ([7],[8],[9]) where any kind of detectable Information such as any visible object or particles and antiparticles could be recreated depending on the fluctuations intensity: Time dimensions are the fields of the QFT. Also Time is described partially in Quantum Electrodynamic QED theory [10] in the sense where virtual particles are the universe neurotransmitters and the path integrals formulations on different paths describes what happen between two neurons (synpases, dendrites paths and neuron soma). In our mathematical models, the path integrals method [11] is used in our model of the Time paths and which is used in Miranker paper [12] for information propagation. The main object of this paper is to give a link between this path integrals model (quantum mechanics) and spacetime curvature (general relativity).

In this paper we will firstly describe the timespace system and signal-Information for both the Brain and the Universe. We will then define our mathematical model of the information diffusion between neurons in the Brain as in the Universe. In section IV we will show how the information aggregated in a neuron is deformed as soon as it arrives in neuron soma and how the total deformation can be seen as the curvature of the Space-Time. Section V explains how from this model we can deduce the model of deformation of space time in the presence of mass and /or energy and hence link between quantum mechanics and general relativily. Finally we will define the different implications of the brain function theory on the universe laws: gravity, quantums mechanics, black holes description,...

\section{SYSTEM DEFINITION OF A REACTION TO AN INCOMING INFORMATION IN BRAIN AND UNIVERSE}

In this section we describe how any Information evolves and may induce a Reaction in the Brain and in the Universe. Any signal-Information (in Universe: particles, objects,... in Brain: reactions, actions... ) evolution must be studied in space and time. But what do we mean by Space and Time? We suppose that Space is where a detectable Information can be seen or in other words any location where any information could exist. In Universe, the Space is the 3 dimensions visible space. For the Brain it is the axonal cone and axon where any Information is recreated to be a potential future reaction.

Time is (are?) the invisible dimension(s) that can be seen in the Information evolution (motions, interactions, changes...). What make the signal-Information evolves in time in the brain ? Synapses and neurotransmitters + neuro-receptors types, dendrites and their structures + neuron soma are the Time components in the Brain. These neuronal components force information to move, to persist, to evolve, to become sometime distorted or to disappear by the inhibitory neurotransmitters effect or because of a insufficient amplitude of the aggregated signal in neuron soma. In fact as soon as an action potential is created in the axonal cone, it is automatically sent to synpases and etc, the Time dimensions of the Brain. The signalInformation propagates in one direction in Time Brain as perhaps a lot of visible objects/particles in Universe. This information inevitably travels in Time but not necessarly in Space. It travels in Time as a signal travels in the synapses + dendrites, and may travel in Space if the same information (deformed or not) is recreated in the neighboring spatial locations (axonal cone) if the amplitude threshold is exceeded.

Before arriving to the neuron soma, a signal-Information has the wave property: it can interfer with itself thanks to spatial and temporal summation of postsynaptics potentials coming from many dendrites and perhaps from many neurons. The aggregated signal has the capacity to be transformed hence to a potential internal or external Reaction if the total amplitude exceeds a particular threshold $(-55 \mathrm{mV}$ in general). Therefore an Information has the wave properties between two linked neurons that share synapses Fig. 1) in Time dimensions, and has a particle properties as soon as it is recreated by summation in the soma the Space dimension. 


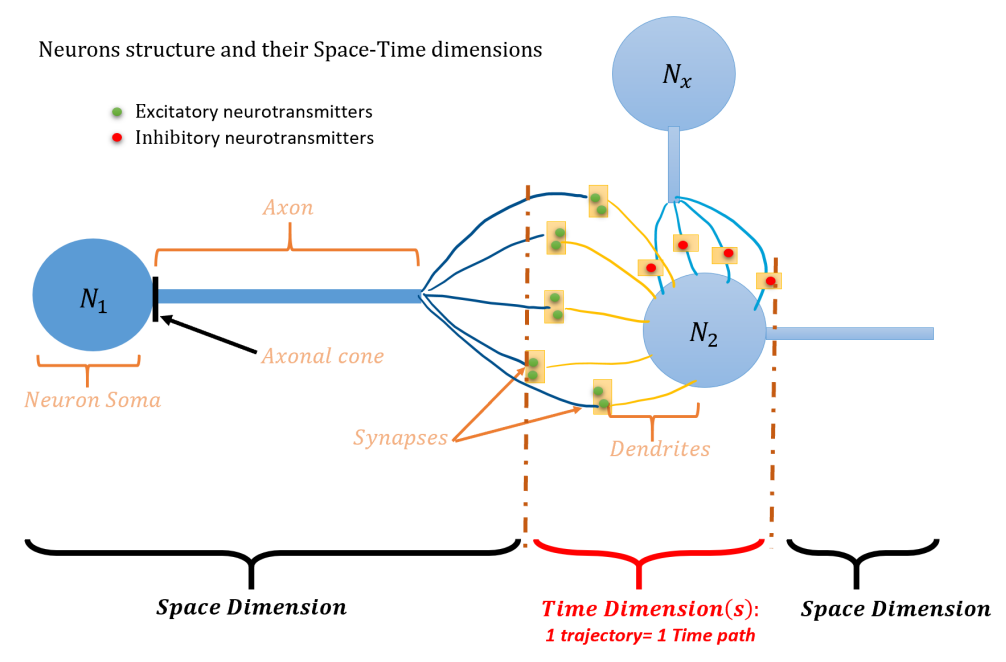

Fig. 1. Neighboring Linked neurons and their Space-Time structure

Particles in quantum mechanics cannot have a particular fixed location or a particular property before its detection: they behave as a wave. But as soon as they are detected they become a normal punctual particle with a known location. This is what happen precisely for human behaviors and decisions. The Reaction to an incoming Information behaves as a particle in quantum mechanics [13]. As an Information arrives to the brain it propagates, interferes with itself thanks to Time components, and as soon as a reaction is needed, there is only one total seen created action: one cannot choose in a restaurant fixed menu both dessert and cheese. But what is this main decided Reaction? The answer is in Le Bihan's work in [14]. In this paper it has been shown experimentally that brain spacetime presents a functional curvature generated by brain activity, in a similar way gravitational masses give our 4-dimensional Universe spacetime its curvature. The heaviest correlated Reaction to the incoming Information will be the decided one. The aim of this paper is to reconcile quantum mechanics and general relativity following these both observations in the Brain and the Universe.

But why there is only one first Reaction? Because a Reaction is not only a set of excitatory neurons activated but also an internal inhibitory Information for other coming correlated reactions thanks to the presence of the inhibitory neurons (synapses with inhibitory neurotransmitters). In fact other correlated in time reactions may follow the first one if the inhibition intensity is not sufficiant: we will continue to cry and/or to act sadely if we are sad until the intensity of crying will decrease a little sadness intensity. Note that some less correlated in time reactions may survive from the inhibitory effect of the existed reactions for long time and could persist beyond the noise effects of the whole day's Information in real life and may exist lately perhapes in dreams.

An incoming Information in the brain is initially processed to a signal-Information, filtered before following particular neural paths. The signal-Information in the brain is therefore a set of action potentials at a particular frequency that its frequency is deformed by particular neurons in the neuronal paths thanks to spatial and temporal summation in neuron soma. The action potential amplitude do not change, it's rather the frequencies of the signals unit that are changed. A final reaction is hence aggregated depending on every human experiences (different neuronal trajectories structure for each human) [15]: The sight of a dog for a given human is not objective. We can see it as a cute little animal and have the need to pet him or as a dangerous animal and run depending on one's past experiences. These two different reactions depend on the Brain SpaceTime evolution. If a dog attacked me before, my heaviest reaction I will have now is to run away. As a simple observer one will not know how the other person would react in presence of a dog if we do not know his history with dogs. The Time dimension in the brain exists and is described by the neuronal paths structures but is uninterpretable for all of us as neurons, synapses, neurotransmitters and etc. are physically same. The difference is just in neuronal path structures precisely in their geometry, their weight in terms of probabilities, and in their neurotransmitters + neuro-receptors types (excitatory or inhibitory...).

In the Universe, An incoming created signal-Information in Space is any particle, object, energy. This Information experiences Time and Space dimensions and undergoes changes in their motions, properties, ... In quantum mechanics, objects have waves and particles properties at the same time. Wave behavior is supposed to be in the Time dimensions and Particle behavior in Space dimension. In this paper as we suppose that Space is the observables dimensions - defined by presence of reel particles-, then Space is where the particle behaves as a punctual particle: this what we really observe or detect. The unobservable part, which has got the wave property is the Time dimensions behavior part. In Time dimensions, particles can interfer with themselves and with one another and as soon as they interact with object present in space a new Information is created: it is the Information of spatial presence of the particle. This new SpaceTime Information is a kind of an Anti-Information in Time dimensions that will inhibit all the most correlated and nearest Time information that 
was propagating freely in Time. Time here is then supposed to be the propagation and the interaction between fields in quantum Vacuum [9] and can be modelled by Path Integrals [11].

Information and Anti-Information in Time dimension are supposed to be the virtual particles and antiparticles created from the quantum Vacuum in the Univers, and are the excitatory neurotransmitters and inhibioty neurotransmittersin the Brain. Elementary particles and Bosons exchanged between particles (Gauge bosons [16]) act similarly as action potentials in the Brain. In fact, a simple presence of a massive Information (an object that has a mass) breaks virtual particles pairs (particles and antiparticles) that exist in the quantum Vacuum. This breaking is done through the intermediary of energy called the Higgs boson. This boson, coming from a real massive particle, mates temporarily to virtual particles and thus change the state of the particles: the virtual becomes real and the real becomes virtual which is inhibited by mating with the detached virtual antiparticle. The Higgs boson is the Information dissiminator of the mass. In other words, Higgs field is the Time field of the mass and Higgs boson is the action potential or the signal-Information unit of the mass of any massive Information. The Higgs boson is the Information unit that brings the Information of the future probably created massive object in the neighboring locations.

Higgs boson and all the other Gauge bosons and the elementary particles are the action potentials of the Information of the universe. Their respective fields and their interactions are the Time dimensions of each type of elementary particle. The virtual particles and antiparticles have similar role than neurotransmitters in neurons. Virtual particles that can supersede reel particles wich are transformed to virtual particles. Excitatory neurotransmitters have the ability to forward the initial Information (deformed or not) to other locations thanks to different time paths aggreagation in this location or to reinforce a particular Information in other particles probably present in this location. If the probability is not sufficient to recreate the object in other locations, the object remains in its past location.

The excitatory neurotransmitters are the virtual particles of the quantum Vacuum that may perpetuate the initial Information in the same location or in other locations, and the virtual antiparticles of quantum Vacuum are the inhibitory neurotransmitters that are the anti-Information that may inhibit the initial Information in the past or inhibit future particular Information.

Thanks to Higgs boson present in the massive Information, this massive Information is never the same during time. Hence any initial Information may also change to other particles sometime even to antiparticles [17], or may just accelerate or decelerate in space etc.

How an Information travels in Time dimensions in Universe ? The answer is in quantum mechanics, QFT and QED that may be described using path integrals. The Time dimension in each field is these different paths that will be explained later in the paper. For the brain, the Paths in the Feynman Path integrals are the trajectories between two linked neurons: synapses with Neurotransmitters + Neuro-receptors and dendrites. The spatial positions are the axonal neuron.

The following table (I) gives a summary on how Information and SpaceTime are defined for both Brain and Universe.

\begin{tabular}{|l||l|l|}
\hline Description & In Brain & In Universe \\
\hline Space & Axonal cone & 3-Dimensions visible space \\
\hline Time & Synapses + dendrites... & $\begin{array}{l}\text { Fields in the Quantum Vacuum [9] and Path } \\
\text { Integrals [11] }\end{array}$ \\
\hline $\begin{array}{l}\text { Information Unit } \\
\text { in Space }\end{array}$ & $\begin{array}{l}\text { Created set of action potentials in Soma thanks to } \\
\text { spatial and temporal summation [5] + Final } \\
\text { Motor-neurons neurotranmistters }\end{array}$ & $\begin{array}{l}\text { Elementary particles in Standard Model [18],[19]: } \\
\text { Particle part in Wave-Particle duality }\end{array}$ \\
\hline $\begin{array}{l}\text { Information Unit } \\
\text { in Time }\end{array}$ & $\begin{array}{l}\text { Interneuron Neurotransmitters + Excitatory and } \\
\text { Inhibitory postsynaptic potential [20] }\end{array}$ & $\begin{array}{l}\text { Virtual Particles + virtual Antiparticles and Bosons } \\
\text { [21] + Wave part in Wave-Particle duality }\end{array}$ \\
\hline \hline
\end{tabular}

TABLE I

INFORMATION AND SPACETIME DEFINITION FOR BRAIN AND UNIVERSE

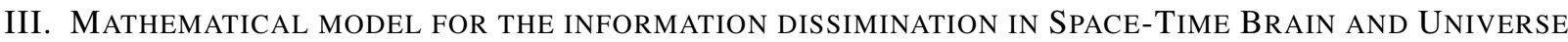

\section{A. Information-Reactions system model in Space-Time dimensions in Brain and for Universe laws}

1) In brain: Between two Brain neurons $N_{1}$ and $N_{2}$ directly linked there are synapses that we will note $s_{12}$. We suppose in our model that there is as much synapses as paths between these two neurons. However the neuron $N_{2}$ can have other synapses (hence other paths) connected to other neighboring neurons $N_{x}$ Fig.1. Moreover from one neuron $N_{1}$ a same information can move to different linked neurons at the same time.

All the signals coming from $N_{1}$ are remodulated by each path and reach the $N_{2}$ neuron soma where they are aggregated according to their arrival spatial and temporal phase thanks to the spatial and temporal additivity property of the 
postsynaptical action potentials in the dendrites. This spatial and temporal summation will ensure interference in the case of time delay between signals coming from any dendrites, or in the case of spatial distance between dendrites incoming to the soma. A same signal-Information coming from $N_{1}$ can be deformed due to different path structures between $N_{1}$ and $N_{2}$ namely by the geometry of paths and their respective neurotransmitters type.

Each neuron $N_{2}$ is activated by the signal transmission aggreagted coming from the linked neuronal neighborhood $N_{x}$. This signal or Information amplitude is denoted by $\psi\left(N_{x}, t\right)$ the wave function of signal neuron $N_{x}$ at time $t$ that may be complex as signals can interfer with one another. If $\psi\left(N_{x_{0}}, t\right)=0$ then there is no signal coming from the neuron $N_{x_{0}}$ at time $t$. When traveling in the $N_{1}$ axon until the collaterals and axonal terminaison, the signal $\psi\left(N_{x}, t\right)$ remains the same

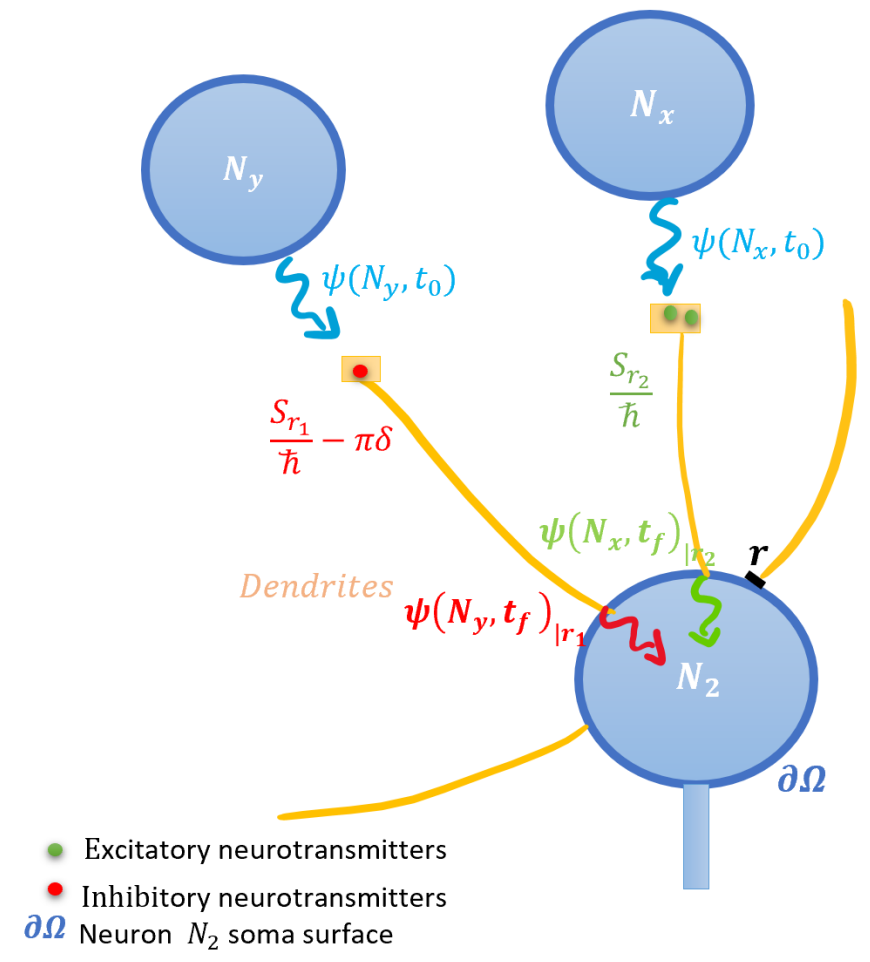

Fig. 2. Signal-Information propagation with Time path action

the only difference is that the signal speed changes along the path due to membrane channel composition and particularly to their diameters. It's known that in the axon (depending on each neuron) the speed of the signal propagation is the highest comparing to the speed in axonal terminaisons and dendrites. As soon as the signal arrives to the synapse, the signal causes the exocytosis of neurotransmitters: the wave is converted into matter which subsequently disappears just after being linked to the neuro-receivers and retransformed to their respective signals: inhibitory signals if the neurotransmitters are inhibitory or excitatory signal if the transmitters are excitatory [22]. The total signal-Information coming to $N_{2}$ is finally deformed due to all these paths components action and also due to paths coming from other correlated neighboring neurons.

The signal-Information from $N_{x}$ to $N_{2}$ in the Brain Time dimension undergoes in each Time path at each time unit $d u$ Fig.3 a normalized action $d \bar{S}$ per unit time defined as following:

$$
\frac{d \bar{S}}{d u}=\frac{L}{\hbar} \Rightarrow \bar{S}=\int \frac{L}{\hbar} d u
$$

$\frac{d \bar{S}}{d u}$ is the action unit of the neurotransmitters in a particular path. $L$ is a path Lagrangian and $\hbar$ is supposed to be a constant (the reduced Planck constant for Universe laws) [23]. $\frac{1}{\hbar}$ acts as the normalization of the energy density in time where if Lagrangian $L$ is equal to one energy unit $L_{1}$ (one action potential for brain) for one time unit $t_{1}$, hence:

$$
\int_{0}^{t_{1}} \frac{L_{1}}{\hbar} d u=1
$$

where $\frac{L_{1}}{\hbar}$ would be equivalent to the probability density to find a quantum of energy at $u$ in probability theory, with $\hbar$ the maximal quantity of energy that can hold a temporal distance unit, the Time distance between two neurons $N_{1}$ and $N_{2}$. With this definition, one can note that $\bar{S}$ is the mean of $\frac{L}{L_{1}}$ which is the number of energy units, or in the brain the number of action potentials at time $u$, or in the Universe laws, is equivalent to the frequency. 
The signal-Information propagating in a particular path with incidence $r$ undergoes a total phase shift of $\overline{S_{r}}$ from $t_{0}$ to $t_{f}$ Fig.2, and the modified signal in this path is:

$$
\psi\left(N_{1}, t_{f}\right)_{\mid r}=\psi\left(N_{1}, t_{0}\right) e^{i \overline{S_{r}}}=\psi\left(N_{1}, t_{0}\right) e^{i \frac{S_{r}}{\hbar}-\delta_{i n h, r, t} \pi}
$$

where $S=\int_{t_{0}}^{t_{f}} L d u$ the path action, and $\delta_{i n h, r, t} \pi$ is equal to $\pi$ if the Information mediator is an inhibitor coming with incidence $r$ FIGURE at time $t$ : an inhibitor neurotransmitter or a virtual antiparticle in Universe. $\delta_{i n h, r, t}=0$ if the mediator is an excitator (particles or excitator neurotransmitters). Adding $-\delta_{i n h, r, t} \pi$ term to the action of a path allows the inhibition of the signal. In fact, if the neurotransmitter in a particular path is inhibitor than $e^{i \frac{S_{r}}{\hbar}-\pi}=-e^{i \frac{S_{r}}{\hbar}}$. $-\delta_{i n h, r, t} \pi$ could be integrated to the matter action of each path, but we will show lately why we consider it all alone Fig.2.

2) In Universe: $\hbar$ can be defined as the maximal quantity of energy that can hold a unity of temporal distance $t_{p}$, the smaller time that two different events can be seen in Space which is the Planck time $t_{p}$ [24]. Moreover, this Planck time is equivalent to a Planck distance $l_{p}$ that can contain a maximum mass equivalent to Planck mass $M_{p}$. The maximal energy, thanks to mass-energy equivalence, is therefore equal to $M_{p} c^{2}$ in the shortest temporal distance $t_{p}$, therefore:

$$
\hbar=M_{p} c^{2} t_{p}=1.0564 \times 10^{-34} \mathrm{~kg} \mathrm{~m}^{2} \mathrm{~s}^{-1}
$$

which is equal to the value of the reduced Planck constant.

Similarly as in the brain, between two any nearest spatial points $N_{1}$ and $N_{2}$ in Universe, with spatial and temporal distance are equivalent respectively to Planck distance $l_{p}$ and Planck time $t_{p}$, there is the quantum Vacuum that we have defined as the Time dimensions in Brain. This Time dimensions are described by paths of path integrals theory where each path has a complex weight $e^{i \frac{S_{r}}{\hbar}-\delta_{i n h, r, t} \pi}$ [25], where $S_{r}$ is the action of the studied path.

A particle in quantum physics is described by the wave function $\psi(N, t)$ that the square module is equal to the probability that the particle is at state $N$ at time $t$, where $N$ can be a space location or can be other states as spin or other quantum property able to modify. In this paper, the study is restricted to the state $N$ of spatial location and therefore the only studied Reaction of a particle to an Information considered here is the spatial motions.

\section{B. Mathematical Model for Information propagation in Space-Time dimensions in Brain/Universe}

At the arrival to the neuron soma, all the signals-information coming from all the dentrites are summed according to their spatial and temporal proximity. This is called the spatial-temporal sum where the signals not far apart temporally and spatially are summed. This property can be written as a spatial and temporal sum on the signals arriving at each point $r$ to the main neuron $N_{2}$ soma. If there is only one incoming dendrite soma therefore there is no spatial shift phase added. The spatial summation of the signal-Information is in neuron soma surface $\partial \Omega$ as the set of connection points of dendrites and neuron forms a spatial surface $\partial \Omega$ Fig.2. The temporal summation is on a time duration $\Delta t$, the time duration that a possible action potential may be created if the amplitude threshold is exceeded. The wave amplitude function of a signal-Information $\psi\left(N_{2}, t_{f}\right)$ in location $N_{2}$ at time $t_{f}$ is therefore:

$$
\psi\left(N_{2}, t_{s}\right)=\int_{\partial \Omega} \int_{\Delta t} \psi\left(N_{r}, t\right) e^{i \frac{S_{r}}{\hbar}-\delta_{i n h, r, t} \pi} D\left[q_{r}(t)\right] d t d r
$$

where $S_{r}$ the action of a path arriving to neuron $N_{2}$ with incidence $r . D\left[q_{r}(t)\right] d t d r$ is the probability measure (density) of the path $q$ arriving at $r$ is the $N_{2}$ neuron soma. $\psi\left(N_{r}, t\right)$ is the initial wave function coming from a neurone $N_{r}$ with the path $q_{r}$ since time $t$.

In the Universe, two any spatial neighboring locations $N_{1}$ and $N_{2}$ are linked by the Vacuum, the Time dimensions which encompasses all quantum fields. A signal-Information in quantum mechanics is described by its wave function vector $\Psi$ in all quantum fields. $\Psi$ here is a wave function vector which each component describes the wave function at each field. One of the unit Information of an object is its mass which is defined by the potential action Higgs boson in its Time dimension the Higgs field. We note $\psi$ the wave function in mass/Higgs field, this is what we will study in this paper. In the rest of the article Higgs or mass boson is the action potential in Time field of mass (Higgs field). The spacetime evolution of the mass boson is described by $\psi(N, t)$, and $|\psi(r, t)|^{2}$ describes the evolution of density of mass Information per time $t$ and space location $r$ (explained later). $\psi(N, t)$ can be modeled samely as (5), where $\Delta t$ the time duration required that the vacuum needs to constantly reconstruct an Information with a particular mass $m, \partial \Omega$ is the surface of the point or the surface of the neuron soma.

Now that an Information may be reconstructed thanks to temporal and spatial summation of action potentials wave functions, we want to know if this Information has been distorted due to different Time paths between two spatial nodes $N_{1}$ and $N_{2}$ and also due to other time paths coming to $N_{2}$ from other spatial nodes $N_{x}$. We will show that the entropy of wave function of paths between $N_{1}$ and $N_{2}$ define the scalar curvature term $\mathcal{R}$ [26] in Hilbert-Einstein action [27], [28]. 


\section{InFOrMATION ENTROPY: BRAIN/UnivERSE SPACETIME CURVATURE}

In this section we want to measure the average level of disorder of the signal-information received by a Neuron $N_{2}$. We introduce here an entropy similar to Shannon entropy in information theory [29]. We recall that Shannon entropy measures the information quantity sent by a information source. The more different information the source emits, the greater the entropy (or uncertainty about what the source emits).

In quantum mechanics, we work with wave functions, a particular probability functions that are complex. Any complex wave function $\psi(r, t)$ can be rewritten as following $\psi(r, t)=R(r, t) e^{i \theta(r, t)}$ with the probability density $p(r, t)=R^{2}(r, t)$ and its phase $\theta(r, t) . \psi(r, t)$ in our study is the wave function of the signal-Information coming to $N_{2}$ at time $t$ from the particular path with extremity $r . p(r, t)=0$ if no signal is coming from the point $r$ at time $t$ in $N_{2}$ or the path with extrimity $r$ does not exist. Nalewajski in [30] gives a definition of the complex entropy by first defining the overall entropy:

$$
s(\psi)=\int-p(r, t)[\ln (p(r, t))+2 i \theta(r, t)] d t d r \equiv s(p)+i s(\theta)
$$

The integral is overall the surface $\partial \Omega$ of the $N_{2}$ neuron soma and over a time duration $\Delta t$.

As all the signal-Information units are supposed similar, the only differences are the frequency of the signal and their probability to arrive to a particular location at time $t$. Hence we introduce a new type of entropy on the phases of signalInformation coming into the neuron $N_{2}$ from each path with extremety $r$ at time $t$. In other words, the entropy that we could take into consideration is the complex part of (6). The spatio-temporal entropy of a signal $\psi$ at time $t_{f}$ in location $N_{2}$ is therefore:

$$
\S\left(\psi\left(N_{2}, t_{f}\right)\right)=\mathcal{L}_{m} \sqrt{|g|}+\int_{\partial \Omega} \int_{\Delta t}-p(r, t) \times(\gamma(r, t)) d t d r
$$

$p(r, t)$ is the density of the incoming signal amplitude per surface and time at $N_{2}$ with incidence $r$ for the signal-Information in the Brain. For the Universe system, in the mass field $p(r, t)$ represents the incoming mass density Information for the signal-Information per time and surface. We do not mean that the mass of an Information is travelling but the signalInformation that cares the mass Information of an object that travels in Time dimensions, namely it could be the wave part of Higgs boson. So if there is no signal-Information present in $N_{r}$, the neuron linked to path with incidence $r$, or there cannot be a Time path between $N_{r}$ and $N_{2}$ hence $p(r, t)=0: p$ encompass the probability of the existence of an initial incoming Information with incidence $r$ and the probability of existence of Time path with incidence $r$. $\gamma(r, t)$ is the spatio-temporal phase shift at time $t$ of the path incoming from $r$ without the matter lagrangian part:

$$
\gamma(r, t)=\frac{S}{\hbar}-\delta_{i n h, r, t} \pi
$$

$S$, or noted before $S_{r}$, is the action on the signal-Information unit in a particular Time path with incidence $r$ that can be defined as following:

$$
\frac{S}{\hbar}=\int^{t} \frac{1}{2} \rho\left(\frac{d q}{d u}\right)^{2} d u .
$$

where $\rho$ represents the mass of the quantum signal-Information in the mass field. In the Brain $\rho$ is the amplitude of a quantum of a signal-Information which is the action potential amplitude (which is always the same $\sim 30 m V$ ). $\frac{d q}{d u}$ is the potential speed of that quantum of Information in the universe or in the brain. $\frac{d q}{d u}$ can be replaced by field formulation.

In the brain, there is no complex interactions between action potentials from different Time paths but only propagation in each path and then summation or inhibition of signals to create or not a new signal in the neuron soma. We can deduce then that $\mathcal{L}_{m} \sqrt{|g|} \sim \int_{\partial \Omega} \int_{\Delta t}-p(r, t) \mathrm{E}_{m} d t d r$ where $\mathrm{七}_{m}$ is the Lagrangian of the initial outgoing signal-Information described by $\psi\left(N_{x}, t_{0}\right)$ from $N_{x}$ Fig.2 where $\psi\left(N_{x}, t_{0}\right)=R(r, t)_{x} e^{i \mathrm{E}_{m}} \cdot \sqrt{|g|}$ represents the volume unit at the studied location which will be explained later, but one can say that its effect is similar to the density $p$.

In the Universe, If the signal-Information is a real particle, its transmitters would be virtual particles (equivalent to excitatory neurotransmitters ) otherwise, its transmitters would be virtual antiparticles (inhibitory neurotransmitters in brain). Particles and antiparticles of the same type have same mass and hence same mass Information (Higgs boson). The difference between the inhibitors and the excitors mediators Lagrangian is the $-\pi$ factor added in the path action explained before. The sign - will be explained later.

In the universe, it is more complicated as the neurotransmitters and action potentials are bosons and/or virtual particles and virtual antiparticles of the quantum fields that may interact one another during Time paths. Universe can be seen as a correlated multi-brains where each quantum field type represents a brain that may interact with one another. Thus $\mathcal{L}_{m}$ represents the Lagrangian density of matter and could be the standard model lagrangian density ([31]).

As $p(r, t)$ represents the incoming mass/amplitude density per surface and time in Universe/Brain, to normalize $p(r, t)$, we multiply and divide by $\frac{M_{p}}{S_{p} t_{p}}$. In fact the maximum of mass quantity that a time path of time Planck $t_{p}$ can carry is 
the mass of Planck $M_{p}$, and $S_{p}$ is the surface of Planck. We consider now $\S\left(\psi\left(N_{2}, t_{f}\right)\right.$ without the Lagragian density of matter:

$$
\S\left(\psi\left(N_{2}, t_{f}\right)\right)=\frac{M_{p}}{S_{p} t_{p}} \int_{\partial \Omega} \int_{\Delta t}-P(r, t) \times \gamma(r, t) d t d r
$$

where $P(r, t)=\frac{p(r, t)}{\frac{M_{p}}{S_{p} t_{p}}}$.

In the neuron soma there is a temporal summation of all the action potentials arrived during $\Delta t$, a duration which after the neuron may create a new action potential or a signal-Information unit that will be sent to neighboring linked neurons with the condition that the sum of signal amplitude exceeds a certain threshold. This duration is the tolerance time that any information unit with respective weight coming from anywhere may be aggregated to create at $N_{2}$ at time $t$ a new information unit: an action potential in Brain.

If the total amplitude exceeds the threshold before achieving duration $\Delta t$, the elementary signal-Information is directly created in Time dimensions.

More explicitely, the entropy, without the Lagrangian of the matter, can be rewritten as follows:

$$
\S\left(\psi\left(N_{2}, t_{f}\right)\right)=\frac{M_{p}}{S_{p} t_{p}} \int_{\partial \Omega} \int_{\Delta t}-P(r, t) \times\left[\frac{1}{\hbar} \int^{t} \frac{1}{2} \rho\left(\frac{d q_{r}}{d u}\right)^{2} d u-\delta_{i n h, r, t} \pi\right] d t d r
$$

hence by normalizing the mass $\rho$ by Planck mass $M_{p}$ (there is a maximum of number of bosons at each Time path that cannot be exceeded in Planck dimensions), we have:

$$
\S\left(\psi\left(N_{2}, t_{f}\right)\right)=\frac{M_{p}}{S_{p} t_{p}} \int_{\partial \Omega} \int_{\Delta t}-P(r, t) \times\left[\frac{M_{p}}{\hbar} \int^{t} \frac{1}{2} \frac{\rho}{M_{p}}\left(\frac{d q_{r}}{d u}\right)^{2} d u-\delta_{i n h, r, t} \pi\right] d t d r
$$

By setting $\frac{M_{p}}{\hbar}$ outside the double integral, we have:

$$
\S\left(\psi\left(N_{2}, t_{f}\right)\right)=\frac{M_{p}^{2}}{S_{p} t_{p} \hbar} \int_{\partial \Omega} \int_{\Delta t}-P(r, t) \times\left[\int^{t} \frac{1}{2} \frac{\rho}{M_{p}}\left(\frac{d q_{r}}{d u}\right)^{2} d u-\frac{\hbar}{M_{p}} \delta_{i n h, r, t} \pi\right] d t d r
$$

$M_{p}, \rho$ etc. have the amplitude significance in the Brain as the mass-Information is not really defined in the Brain.

$-P(r, t)\left[\int_{t} \frac{1}{2} \frac{\rho}{M_{p}}\left(\frac{d q_{r}}{d u}\right)^{2} d u-\delta_{i n h, r, t} \pi\right]$ represents the variation in time of spatio-temporal square distance that a signalInformation in Universe or in Brain travels from two nearest points $N_{1}$ to point $N_{2}$ taking the path with an incidence $r$ in $N_{2}$ at time $t_{2}$ Fig.3. We note the variation in time of square distance of the particular Time path with incidence $r$ without the inhibition part $\frac{\partial g_{x_{r}, t}}{\partial t}$ :

$$
\frac{\partial g_{x_{r}, t}}{\partial t}=-P(r, t)\left[\int_{t_{1}}^{t} \frac{\rho}{M_{p}}\left(\frac{d q_{r}}{d u}\right)^{2} d u\right]
$$

and the variation in time of square distance of the inhibitor Time path with incidence $r \frac{\partial \bar{g}_{x_{r}, t}}{\partial t}$ where:

$$
\frac{\partial \bar{g}_{x_{r}, t}}{\partial t}=P(r, t) \frac{\hbar}{M_{p}} \delta_{i n h, r, t} \pi
$$

The distance between two points $N_{1}$ and $N_{2}$ is infinite if no Information can propagate between them i.e. if $P(r, t)=0$ for all Time incidence $r$ at $N_{2}$. $\frac{\partial g_{x_{r}, t}}{\partial t}$ is surely to be negative if the mediators are excitors (as $\delta_{i n h, r, t} \pi=0$ ) and as the mass $\rho$ of a boson or a virtual particle is positive as $\left(\frac{d q_{r}}{d u}\right)^{2}$. This means that the distance between the two neurons is decreasing between times $t_{1}$ and $t$ if $P(r, t)$ is stricly positive i.e. the path exists, virtual particles/excitatory transmitters exist and the signal-Information exist.

However if the mediators are inhibitors then $-\delta_{i n h, r, t} \pi=-\pi$ and this distance is getting greater than the previous one as $\frac{\partial \bar{g}_{x_{r}, t}}{\partial t}$ would be always positive. This leads to a kind of a virtual spatio-temporal expansion between the two nearest points $N_{1}$ and $N_{2}$. We rewrite the mass entropy or the Information entropy:

$$
\left.\S\left(\psi\left(N_{2}, t_{f}\right)\right)\right)=\frac{M_{p}^{2}}{S_{p} t_{p} \hbar}\left[\frac{1}{2} \int_{\partial \Omega} \int_{\Delta t} \frac{\partial g_{x_{r}, t}}{\partial t} d t d r+\frac{\hbar}{M_{p}} \int_{\partial \Omega} \int_{\Delta t} P(r, t) \delta_{i n h, r, t} \pi d t d r\right]
$$

We consider now :

$$
\begin{aligned}
-\vec{n} d S & =\text { dr surface unit } \\
-\vec{n} & =\text { incoming normal vector to } d S \\
\vec{F} & =\overrightarrow{\operatorname{grad}} \frac{\partial g_{x_{r}, t}}{\partial t} \\
\vec{\Pi} & =\overrightarrow{\operatorname{grad}}\left(\frac{\hbar}{M_{p}} P(r, t) \delta_{i n h, r, t} \pi\right)=\overrightarrow{\operatorname{grad}} \frac{\partial \bar{g}_{x_{r}, t}}{\partial t}
\end{aligned}
$$


where $\vec{\Pi}$ can be rewritten as follows:

$$
\vec{\Pi}=\frac{\hbar}{M_{p}} \pi P(r, t) \overrightarrow{\operatorname{grad}}\left(\delta_{i n h, r, t}\right)
$$

Hence the scalar product of $\vec{n} d S$ and $\vec{\Pi}$ :

$$
-\vec{n} d S . \int^{t} \vec{\Pi} d u=-\frac{\hbar}{M_{p}} \pi P(r, t) \delta_{i n h, r, t} d S
$$

$\vec{F}$ is the resulted vector of a particular path which narrows the distance between $N_{1}$ and $N_{2}$ neurons and $\vec{\Pi}$ is the vector that increases this distance. One can note that to $N_{2}$ the distance is identical to any direct neighboring neuron $N_{x}$ as the distance is only the sum of all these vectors from any incoming path to $N_{2}$ Fig.3. The distance between two any linked neighboring points $N_{x}$ and $N_{2}$ depends on the Time paths and on the initial incoming signal-Information. Space and Time are hence relative depending on what is going on between the nearest two spatial points and on the incoming/present signal-Information. The importance of the sign - next to $\pi$ in the inhibitory part choice is materialized here: in the wave
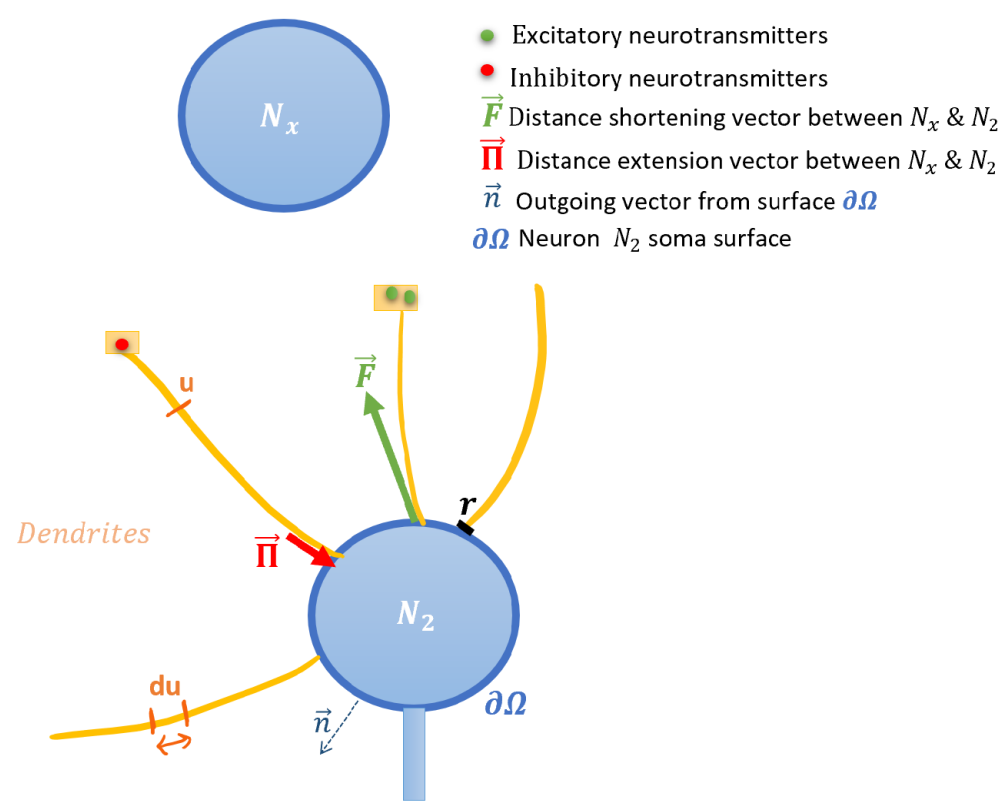

Fig. 3. Creation of the distance between two linked points $N_{x}$ and $N_{2}$

function $\psi$ it has no impact but in signal-Information flow at point $N_{2}$ it precises that the Information is removed from $N_{2}$ which leads to a probable lengthening of the distance between two neighboring points for the studied signal-Information.

The scalar product $\vec{F} \cdot \vec{n} d S$ is equal to $P(r, t) \frac{\rho}{M_{p}}\left(\frac{d q_{r}}{d u}\right)^{2} d r$, therefore the Mass-Information entropy in Universe or the signal-Information entropy in the Universe and in the Brain thanks to Gradient theorem is:

$$
\S\left(\psi\left(N_{2}, t_{f}\right)\right)=\frac{M_{p}^{2}}{S_{p} t_{p} \hbar}\left[\frac{1}{2} \int_{\partial \Omega} \int_{\Delta t} \int^{t}-\vec{F} \cdot \vec{n} d u d t d S-\frac{\hbar \pi}{M_{p}} \int_{\partial \Omega} \int_{\Delta t} P(r, t) \delta_{i n h, r, t} d t d S\right]
$$

Thanks to Green-Ostragradski theorem for Riemannian manifold in the first integral, by interverting integrals and by replacing in the second integral $d u$ by $\frac{1}{c} \times c d u$ and hence $d V=c d u \times d S$, we have:

$$
\left.\S\left(\psi\left(N_{2}, t_{f}\right)\right)=\frac{M_{p}^{2}}{S_{p} t_{p} \hbar}\left[\frac{1}{2} \int_{\Omega} \int_{\Delta t} \int^{t}-\operatorname{div}(\vec{F}) d u d t d V-\frac{\hbar \pi}{M_{p} c} \int_{\Delta t} \int_{\Omega} P(r, t) \overrightarrow{\operatorname{grad}}\left(\delta_{i n h, r, t}\right)\right) d V d t\right]
$$

By interverting div and $\frac{\partial}{\partial t}$ and integrating in time in the first integral, we have:

$$
\S\left(\psi\left(N_{2}, t_{f}\right)\right)=\frac{M_{p}^{2}}{S_{p} t_{p} \hbar}\left[\frac{1}{2} \int_{\Omega} \int^{t}-\operatorname{div}(\overrightarrow{\operatorname{grad}}(g)) d u d V-\frac{\hbar \pi}{M_{p} c} \int_{\Delta t} \int_{\Omega} \delta_{i n h, r, t} P(r, t) d V d t\right]
$$

One can see that the term in second integral can be assimilable to another term as follows: $\int_{\Delta t} \int_{\Omega} \delta_{\text {inh }, r, t} P(r, t) d V d t \sim$ $\frac{1}{4 \pi} l_{p}{ }^{3} \sqrt{|g|}$, ( where $\sim$ is the order of) as $P(r, t)$ is the mass Information probability of arriving from path with incidence $r$ at time $t$ where $\sqrt{|g|}$ is the 4 dimensional volume deformed by $P \cdot \frac{1}{4 \pi \Delta t}$ would represent the density of inhibitors and 
$l_{p}{ }^{3} \Delta t$ the spacetime volume unit. Hence the second integral of (18) that we will note $B$, by normalizing $d V$ with $d v=\frac{d V}{l_{p}{ }^{3}}$, is:

$$
\left.B=\frac{\hbar}{M_{p} c} \pi \int_{\Omega} \int_{\Delta t} \delta_{i n h, r, t} P(r, t)\right) d t d v \sim \frac{\hbar \pi}{M_{p} 4 \pi c} \sqrt{|g|} \simeq 2.02567 \times 10^{-35} \sqrt{|g|}
$$

Which is quite representative of the cosmological constant value $\Lambda$ in time metric [32]. However, we want to compute the second integral noted $B$ in (19) similarly as we have done for the first integral:

$$
\S\left(\psi\left(N_{2}, t_{f}\right)\right)=\frac{M_{p}^{2}}{S_{p} t_{p} \hbar}\left[\frac{1}{2} \int_{\Omega} \int^{t}-(\Delta g) d u d V-\int_{\Omega} \int^{t}(\Delta \bar{g}) d u d V\right]
$$

Therefore we have the second integral equivalent to:

$$
B=-\int_{\Omega} \int^{t}(\Delta \bar{g}) d u d V \sim-\Lambda l_{p}^{3} \sqrt{|g|}
$$

By considering the approximation $R i c \simeq-\frac{1}{2} \Delta g$ in the first integral noted $A$ of (18), where Ric is the Ricci curvature, given in local coordinates by $R_{i j}=(R i c)_{i j}$ so that Ric is a trace of the Riemann curvature, hence:

$$
A \simeq \frac{M_{p}^{2}}{S_{p} t_{p} \hbar} \int_{\Omega}^{t} \operatorname{Ric} d u d V
$$

By normalyzing $d V$ with $l_{p}^{3}$ the Planck volume we have:

$$
\S\left(\psi\left(N_{2}, t_{f}\right)\right) \simeq \frac{M_{p}^{2} l_{p}^{3}}{S_{p} t_{p} \hbar}\left[\int_{\Omega} \int^{t} \operatorname{Ric} d u d v-\Lambda \sqrt{|g|}\right]
$$

where $d v=\frac{d V}{l_{p}}$ is the normalized volume unit. Using another time Ostrogradski theorem in the integral :

$$
\S\left(\psi\left(N_{2}, t_{f}\right)\right) \simeq \frac{M_{p}^{2} l_{p}^{3}}{S_{p} t_{p} \hbar}\left[\int_{\nu} \int^{t} \operatorname{div}(\operatorname{Ric}) d u d \nu-\Lambda \sqrt{|g|}\right]
$$

where $d \nu=d v \times d t$. And from the contracted second bianchi identity where $R$ is the scalar curvature, we have $\operatorname{div}($ Ric $)=\frac{1}{2} d_{u} R$ hence:

$$
\int_{\nu} \int^{t} \operatorname{div}(\operatorname{Ric}) d u d \nu=\frac{1}{2} \int_{\nu} \int^{t} d_{u} R d u d \nu \sim \frac{\mathcal{R}}{2} \sqrt{|g|}
$$

where $\mathcal{R}$ is the scalar curvature of Einstein equation in general relativity. Finally we deduce that:

$$
\S\left(\psi\left(N_{2}, t_{f}\right)\right) \simeq \frac{M_{p}^{2} l_{p}^{3}}{S_{p} t_{p} \hbar}\left(\frac{\mathcal{R}}{2}-\Lambda\right) \sqrt{|g|}
$$

Integring finally $-k . r$ to $\mathcal{R}$ ensures that the scalar curvature is null in the empty space case. From Planck units where $G$ is the gravitational constant, we have:

we can deduce that:

$$
\begin{aligned}
M_{p} & =\sqrt{\frac{c \hbar}{8 \pi G}} \\
S_{p} & =l_{p}^{2} \\
c & =\frac{l_{p}}{t_{p}}
\end{aligned}
$$

where $\kappa$ the Einstein gravitational constant in time metric [33], as In Einstein's original publication, the choice is $\kappa=\frac{8 \pi G}{c^{2}}$, in which case the stress-energy tensor components have units of mass density. Therefore adding the matter effect:

$$
\S\left(\psi\left(N_{2}, t_{f}\right)\right) \sim \frac{1}{\kappa}\left(\frac{\mathcal{R}}{2}-\Lambda\right) \sqrt{|g|}+\mathcal{L}_{m} \sqrt{|g|}
$$

Which is the Lagrangian density of the Einstein-Hilbert action.

We finally can conclude that the entropy of the signal-Information at any point represent the scalar curvature and an approximation of the cosmological constant. Hence the cosmological constant could define the antimatter that had disappeared since the Big Bang. The gravity could be assimilated to the degree of correlation between massive objects and not a kind of interaction. A highly massive object $\mathbf{B}$ or signal-Information impacts highly the Time field of any other 
closed correlated signal-Information $\mathbf{C}$ (less massive) as Time paths coming from particles of $\mathbf{B}$ are added to the paths of the studied object $\mathbf{C}$ Fig.4. Hence the probability of $\mathbf{C}$ object existence is much higher next to the object $\mathbf{B}$ at time $t+1$ than its past location at time $t$ : this is the gravity effect. Finally we conclude that the entropy due to the term probability $P$,

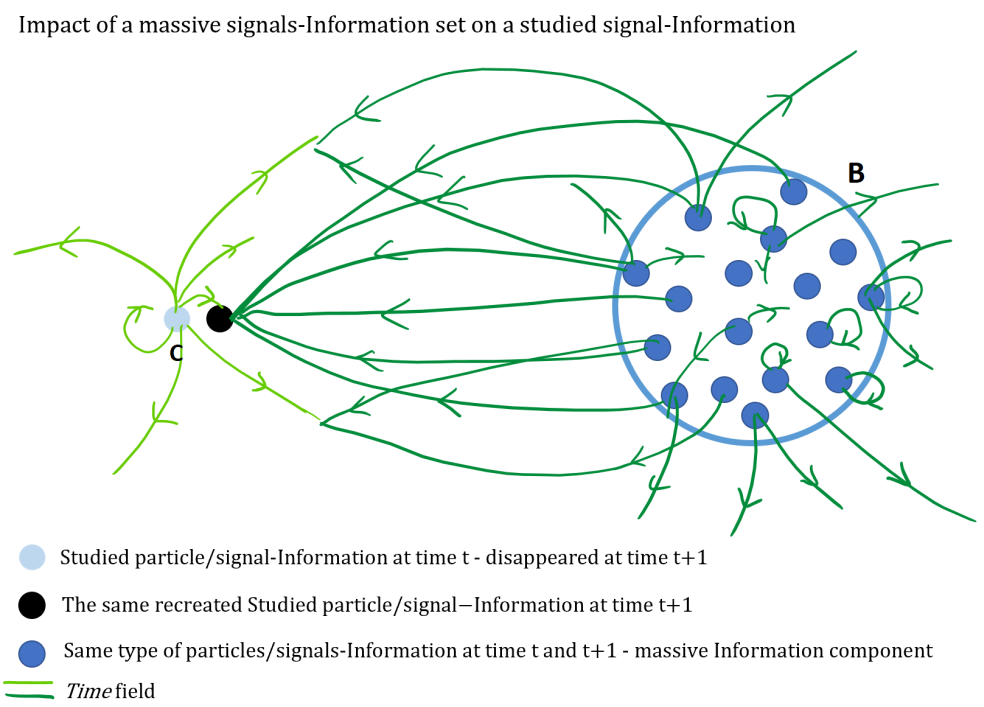

Fig. 4. Impact of a massive set of signals-Information on another studied Information

wich encompass the existence of the signal-Information (massive object in gravity) and the possible existence of Time paths between any spacetime points, is the gravity impact of the massive objects. In the Brain, the gravity effect is seen in the Final Reaction to an incoming Information: The decided Reaction is the most correlated one to the incoming Information.

\section{IMPLICATIONS ON UNIVERSE LAWS AND HUMAN BEHAVIORS}

Gravity: In universe, Gravity is the result of massive matter correlation, and the spacetime curvature is the massive Information entropy created by the neighboring massive objects. In the brain it is the same, an incoming information excites the most correlated and heaviest action i.e. neurons that have the most number of Time paths linked to the initial activated neurons.

Black Holes: There is no more correlation between objects, even the information with itself. In fact all any possible Time path is absorbed by the massive point inside the black hole. No such quantum fields could exist: the information is detructed as no Time path could exist in black holes. There has been a tearing of quantum fields as any probable created field is absorbed by the massive point. In the brain it is just like a neuron that its synapses or dendrites are destructed, any incoming information to these type of neurons is lost and no action related to these neurons can be created.

Universe expansion: This could be a result of the disappeared antimatter since the Big Bang that could be present between two set of matters and their fields. In the brain it is similar to two set of excitatory neurons which one is an inhibitor to the other one and reciprocally.

The Heisenberg incertitude principle $\Delta x \Delta p \geq \frac{\hbar}{2}$ : This formula may be explained as following: if we try to locate a particle in a particular position then we give a high intensity of Information concentrated into this location which may enhance the density of Time paths from this location to this same location. We have inhibited the other paths to other locations and we have enhanced at the same time the energy of the particle localization. It is what happens in the brain when an Information with very high amplitude is coming: it propagates with high probability in different neuronal paths and hence the reaction is difficult to be known.

Quantum decoherence: The presence of inhibitory neurons i.e. with inhibitory neurotransmitters ensures the quantum decoherence of later reactions to an incoming Information. As the inhibitory particles in Universe are supposed to be virtual antiparticles, they could have the same impact by inhibiting other Reactions that may exist at time $t+1$ : they inhibit the possible particles next to the real created one in space and time (even in the past).

\section{REFERENCES}

[1] Roberts Bartholow. Art. i.-experimental investigations into the functions of the human brain. The American Journal of the Medical Sciences (1827-1924), (134):305, 1874.

[2] Kevin J Bender and Laurence O Trussell. The physiology of the axon initial segment. Annual review of neuroscience, 35:249-265, 2012. 
[3] Alan L Hodgkin and Andrew F Huxley. Action potentials recorded from inside a nerve fibre. Nature, 144(3651):710-711, 1939.

[4] Jiang Hao, Xu-dong Wang, Yang Dan, Mu-ming Poo, and Xiao-hui Zhang. An arithmetic rule for spatial summation of excitatory and inhibitory inputs in pyramidal neurons. Proceedings of the National Academy of Sciences, 106(51):21906-21911, 2009.

[5] Anthony CC Coolen, Reimer Kühn, and Peter Sollich. Theory of neural information processing systems. OUP Oxford, 2005.

[6] Bryan Kolb, Ian Q Whishaw, and G Campbell Teskey. An introduction to brain and behavior. Worth New York, 2001.

[7] Lewis H Ryder. Quantum field theory. Cambridge university press, 1996.

[8] Tom Lancaster and Stephen J Blundell. Quantum field theory for the gifted amateur. OUP Oxford, 2014.

[9] Peter W Milonni. The quantum vacuum: an introduction to quantum electrodynamics. Academic press, 2013.

[10] Richard P Feynman. Quantum electrodynamics, volume 3. Westview Press, 1998.

[11] Richard P Feynman, Albert R Hibbs, and Daniel F Styer. Quantum mechanics and path integrals. Courier Corporation, 2010.

[12] Willard L Miranker. Path integrals of information. Yale Univ. DCS/TR-1215, 2002.

[13] Jerome R Busemeyer and Peter D Bruza. Quantum models of cognition and decision. Cambridge University Press, 2012.

[14] Denis Le Bihan. On time and space in the brain: A relativistic pseudo-diffusion framework. Brain Multiphysics, 1:100016, 2020.

[15] Hind Zaaraoui. An introduction to the emotional impact modeling method of an event. PsyArXiv, 2020.

[16] John Owen Edward Clark. The essential dictionary of science. Barnes \& Noble, 2004.

[17] LHCb collaboration. Observation of the mass difference between neutral charm-meson eigenstates. Technical report, CERN, Geneva, Jun 2021. All figures and tables, along with machine-readable versions and any supplementary material and additional information, are available at https://cern.ch/lhcbproject/Publications/p/LHCb-PAPER-2021-009.html (LHCb public pages).

[18] David Griffiths. Introduction to elementary particles. John Wiley \& Sons, 2020.

[19] Juerg Beringer, JF Arguin, RM Barnett, K Copic, O Dahl, DE Groom, CJ Lin, J Lys, H Murayama, CG Wohl, et al. Review of particle physics. Physical Review D-Particles, Fields, Gravitation and Cosmology, 86(1):010001, 2012.

[20] Hiroshi Takagi. Roles of ion channels in epsp integration at neuronal dendrites. Neuroscience research, 37(3):167-171, 2000.

[21] Mark Thomson. Modern particle physics. Cambridge University Press, 2013.

[22] Harvey Lodish, Arnold Berk, S Lawrence Zipursky, Paul Matsudaira, David Baltimore, and James Darnell. Neurotransmitters, synapses, and impulse transmission. In Molecular Cell Biology. 4th edition. WH Freeman, 2000.

[23] Richard P Feynman. The principle of least action in quantum mechanics. In Feynman's Thesis-A New Approach To Quantum Theory, pages 1-69. World Scientific, 2005.

[24] National Institute of Standards and Technology. NIST Physics Laboratory. The NIST Reference on Constants, Units and Uncertainty. National Institute of standards and technology, 1998.

[25] Paul AM Dirac. The lagrangian in quantum mechanics. In Feynman's Thesis-A New Approach To Quantum Theory, pages 111-119. World Scientific, 2005.

[26] Arthur L Besse. Einstein manifolds. Springer Science \& Business Media, 2007.

[27] Richard Feynman. Feynman lectures on gravitation. CRC Press, 2018.

[28] Charles W Misner, Kip S Thorne, and John Archibald Wheeler. Gravitation. Macmillan, 1973.

[29] Claude E Shannon. A mathematical theory of communication. The Bell system technical journal, 27(3):379-423, 1948.

[30] Roman F Nalewajski. Complex entropy and resultant information measures. Journal of Mathematical Chemistry, 54(9):1777-1782, 2016.

[31] Bohdan Grzadkowski, M Iskrzyński, Mikolaj Misiak, and Janusz Rosiek. Dimension-six terms in the standard model lagrangian. Journal of High Energy Physics, 2010(10):85, 2010.

[32] Moshe Carmeli and Tanya Kuzmenko. Value of the cosmological constant: Theory versus experiment. In AIP Conference Proceedings, volume 586, pages 316-318. American Institute of Physics, 2001.

[33] Ronald Adler, Maurice Bazin, and Menahem Schiffer. Introduction to general relativity, volume 220. McGraw-Hill New York, 1975. 